\title{
Comparison of Calculated and Experimental Cascade Performance for Controlled-Diffusion Compressor Stator Blading
}

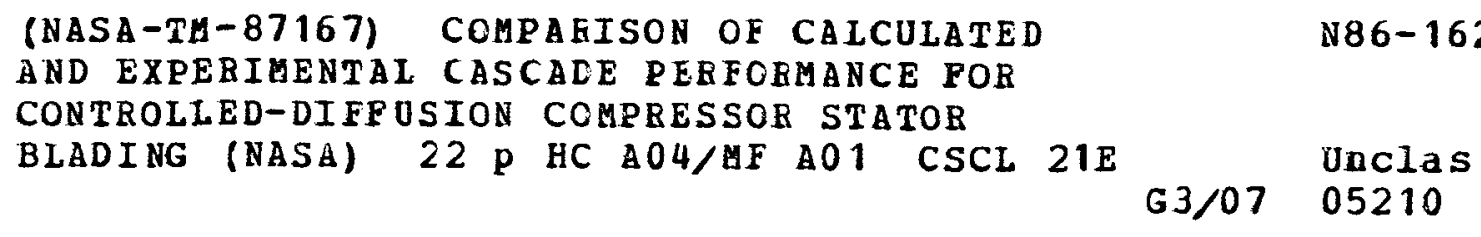

Nelson L. Sanger

Lewis Research Center

Cleveland, Ohio

and

Raymond P. Shreeve

Navel Postgraduate School

Monterey, California

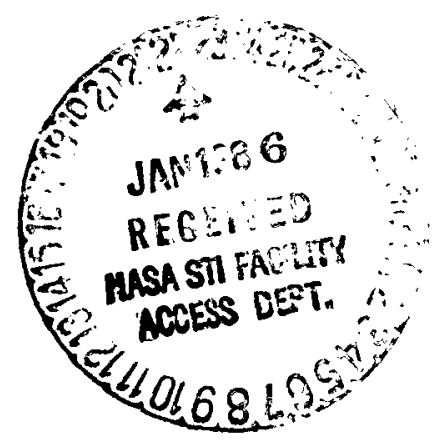

Prepared for the

International Gas Turbine Conference and Exhibit

sponsored by the American Society of Mechanical Engineers

Dusseldorf, West Germany, June 8-12, 1986

\section{N/Sก}




\title{
$\therefore \div$ \\ COMPARISON OF CALCULATED AND EXPERIMENTAL CASCADE PERFORMANCE FOR CONTROLLED-DIFFUSION COMPRESSOR STATOR BLADING
}

\author{
Nelson L. Sanger \\ National Aeronautics and Space Administration \\ Lewis Research Center
}

Cleveland, Ohio 44135

and

Raymond $P$. Shreeve

Naval Postgraduate School

Turoopropulsion Laboratory

Monterey, California 93940

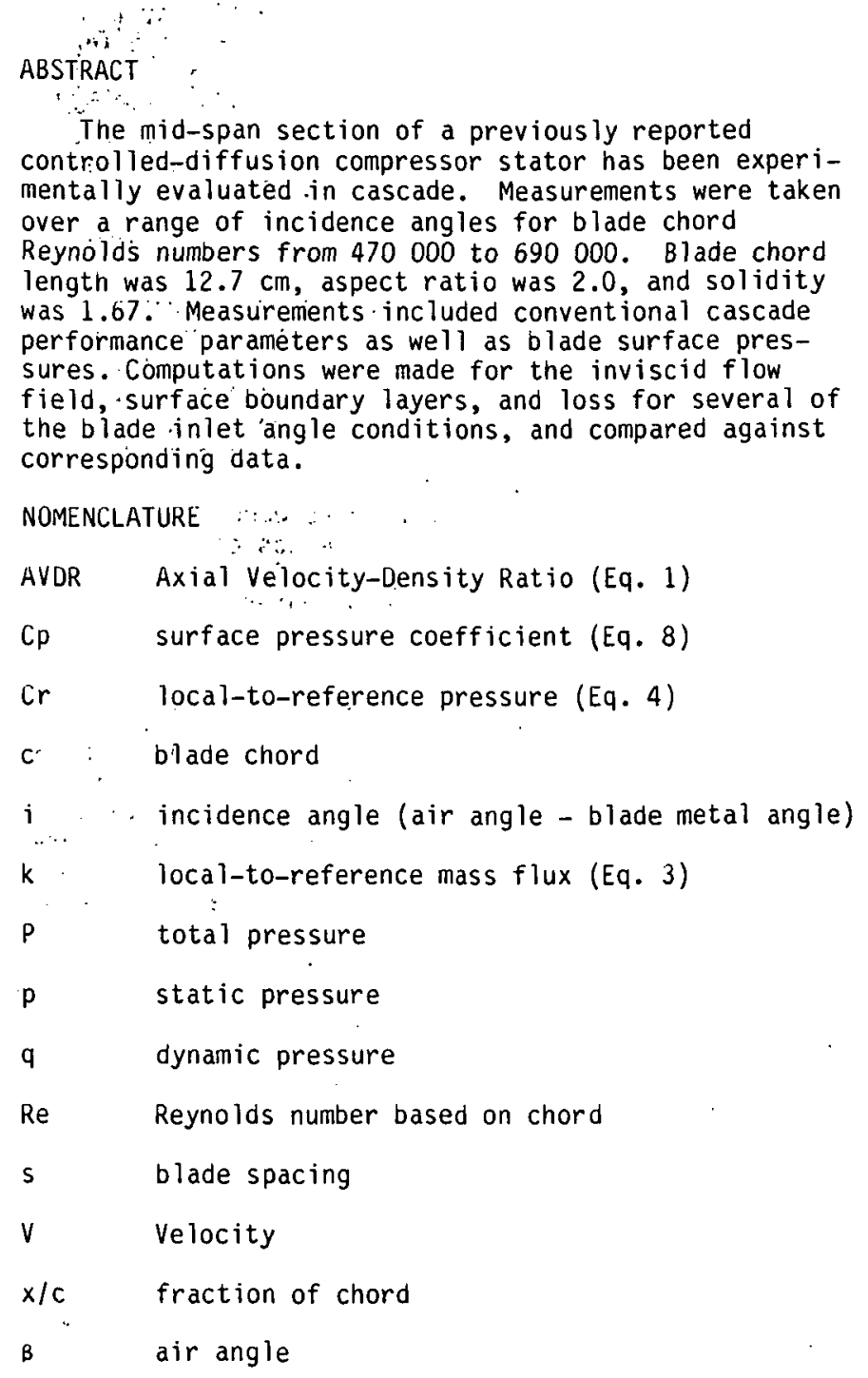

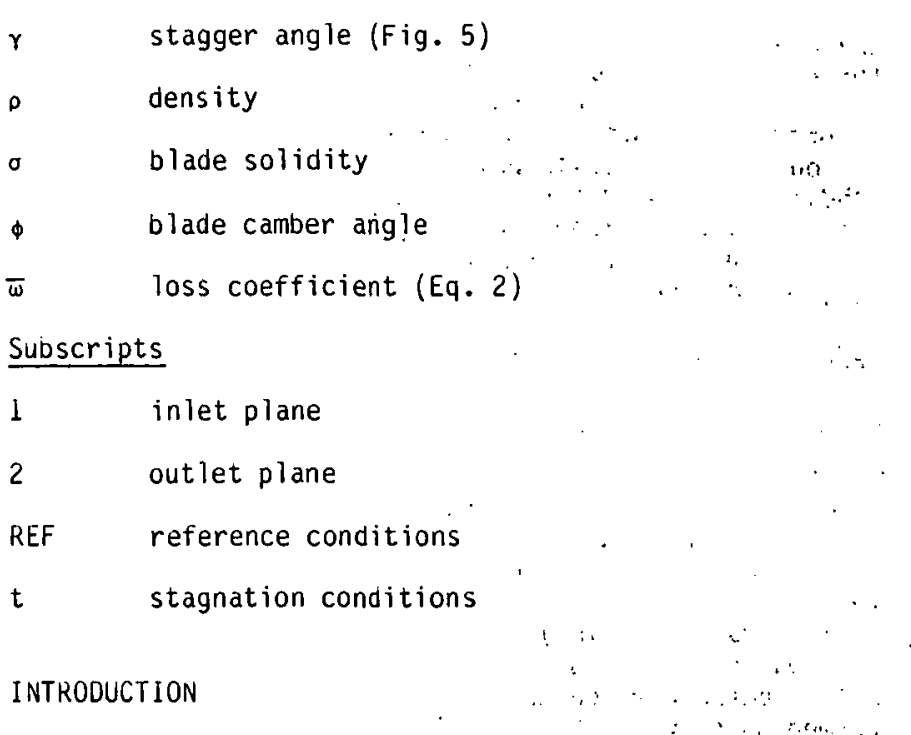

In a paper presented at a recent International Gas. Turbine Conference, the design of a controlled diffusion compressor stator using an automated design procedure based on numerical optimization was described (1). The controlled diffusion stator was a redesign of the first stage stator of the NASA Two-Stage Fan (2). The original design was hignly successful, demonstrating a first stage peak adiabatic efficiency of 87 percent, and a radial distribution of loss across the stator which was. remarkably low. The purpose of the redesign was to develop and demonstrate the feasibility of an automated design process using numerical optimization methods. Although significant performance improvement from an already excellent stator design was not expected, at least equivalent performance was expected. In addition, the redesigned stator was intended to provide a research vehicle for the new controlled diffusion class of olading.

As described in Ref. 1, the controlled diffusion stator was designed using a series of computational analysis methods coupled by a numerical optimization procedure. The blade snapes were specified by a 
geometry code extracted from the NASA throughflow compressor design program (3). Blade section mean lines and thickness distributions were described by several polynomial curves. The potential flow about each two dimensional blade section was calculated by the TSONIC code developed by Katsanis (4). Surface boundary layers were calculated using the MCNally BLAYER code (5), and the optimization procedure used to couple all. of the aerodynamic and geometric codes together was the COPES/CONMIN code $(6,7)$.

The mid-span section of the blade has been built and tested in a cascade wind-tunnel at the Naval Postgraduate School. Although inlet Mach numbers (0.2) in this subsonic tunnel are less than the design level (0.68), experiments can be conducted at realistic Reynolds numbers $(500000$ to 700000$)$ on relatively large scale blading (12.7 cm chord), on which detailed surface pressures can be obtained. Furthermore, since the computational codes used to design the blading were quasi-three-dimensional and steady state, it is of interest to obtain experimental data in a similar flow environment, as well as in the real, unsteady, fully tnree-dimensional environment of the compressor stage. The data obtained in cascade provide an excellent opportunity to assess the accuracy of the flow codes not only at the design point, but also at off-design conditions. In this report, data obtained over the full range of incidence angle (including flow visualization data), are compared against the calculated results from several flow codes.

\section{EXPERIMENTAL PROGRAM}

\section{Description of the Test Facility}

The arrangement of the cascade wind tunnel facility is snown in Fig. 1. The tunnel is supplied by a $700 \mathrm{HP}$ blower in the basement of the building through an arrangement of sound baffles and turning vanes. The air enters the tunnel through two, two-dimensional bel1mouth contractions in series which produce a 152.4 by $25.4 \mathrm{~cm}$ ( 60 by 10 in.) section just above floor leve.1. The $25.4 \mathrm{~cm}$ width is maintained between heavy side walls, between which an adjustable test section is formed as shown in Fig. 2. The blades of the test cascade are mounted in a rack which can be moved along the (fixea) back wall. The front wall is readily removed by overhead crane to give access to test section and instrumentation (Fig. 3 ). The air angle into the test cascade is governed by the setting of the adjustable lower end walls and the 59 inlet guide (or turning) vanes. The adjustable upper (exit) end walls are individually adjusted during operation to angles required to produce uniform static pressure at the wall in the blade-to-blade direction. Static pressure taps are provided at $5.08 \mathrm{~cm}$ intervals in the Dlade-to-blade direction at vertical (axial) distances of $41.28 \mathrm{~cm}$ upstream and $16.51 \mathrm{~cm}$ downstream of the center of the test blades. Spanwise and blade-to-blade probe traverses are provided at $31.12 \mathrm{~cm}$ vertically upstream and $27.31 \mathrm{~cm}$ vertically downstream of the same reference. Plexiglass windows in the removable wall at the test blading facilitate the use of flow visualization techniques (Fig. 4).

Unusual features of the facility are the large scale, the large number of test blades $(20$, at $7.62 \mathrm{~cm}$ spacing) and the technique for controlling inlet air angle. Considerable facility development and test experience (8-13) preceded the present test program. Upstream flow uniformity, periodicity and steadiness, and acceptable axial velocity-density values without resort to boundary layer suction, were achieved as a result of the initial design features and subsequent experimental programs. Turbulence intensity, measured across five turning vane pitches at blade mid-height, was 2 percent.

\section{Test Blade}

The blade shape is represented in cascade in Fig. 5. The meanline is represented by two segments, each described by polynomial curves. A symmetrical thickness distribution is built on the meanline. This thickness is made up of a front segment and a rear segment, each represented by a separate polynomial. The polynomial coefficients were arrived at through an optimization process described in Ref. 1. A summary of the section geometrical properties is given in Table $I$.

\section{Instrumentation and Data Acquisition}

The instrumentation is summarized in Table II. The flow survey probes were calibrated using Zebner's surface approximation method (Ref. 15) with computer programs given in Ref. 16.

A Hewlett-Packard HP3052 Data Acquisition system incorporating an HP9845A desktop computer and NPS/TPL HG-78K Scanivalve Controller were used to acquire pressure and temperature data. Flow survey probes and tunnel reference pressures were connected to one 48 port Scanivalve and blade surface pressures and reference readings to another. Measurement uncertainties are given in Table III. Tunnel wall statics were displayed and observed on a 50 tube water manometer.

The china clay technique was used as described in Ref. 13. Surface patterns were recorded in color using a movie camera, and transition and separation.distances were scaled from projected frarnes. The observations : made with china clay were first 'calibrated.' by applications of the technique to botn a sharp and a rounded leading edge flat $p$ late model set into the cascade in place of the test blading (Ref. 13).

Turbulence intensity measurements were taken with a full inicron tungsten wire. The wire was calibrated using King's Law over fourteen points from a tunne? dynamic pressure of 0 to $39.4 \mathrm{~cm}$ of water. The correlation coefficient was 0.995 .

\section{Testing Procedure and Data Reduction}

Seven inlet air flow angles from 24 to $46^{\circ}$ were selected and set in turn. At each angle setting similar procedures were followed. With the tunnel set to the desired inlet dynamic pressure (either 33 or $20 \mathrm{~cm}$ of water) tail boards and IGVs were adjusted to give nearuniform wall static pressures both upstream and downstream of the test blading. Blade-to-blade probe surveys were then conducted over four adjacent blade spaces about the center blade. Probe data and reference data were recorded at $0.64 \mathrm{~cm}$ intervals over the outer spaces, and $0.25 \mathrm{~cm}$ intervals over the central spaces. The lower probe trailed the upper by $3.84 \mathrm{~cm}$ throughout the traverse procedure. A spanwise probe survey was then conducted at $1.27 \mathrm{~cm}$ intervals at the upper station with the probe located at about $2.5 \mathrm{~cm}$ from the suction side of the center blade. The survey procedure was repeated with the lower probe at approximately $2.5 \mathrm{~cm}$ from the pressure side of the central blade. The blade surface pressures and reference data were then recorded witn tunnel reference data.

China clay observations were made for similar settings of the cascade at a dynamic pressure of approximately $9.3 \mathrm{~cm}$ of water. The somewhat reduced velocity was necessary since higher velocities give too rapid a drying rate, and difficulty in recording and interpreting tne drying patterns. Blade static pressure distributions were recorded when china clay observations were made. These data could be compared with data already recorded at two higher Reynolds Number. 
In the reduction of the probe survey data, after reduction to velocity, pressure and flow angle at each point, the data were referenced to quantities derived from the tunnel plenum to atmospheric pressure (driving potential). As shown in Ref. 9 and 10, this permits integration of the measurements over the blade-to-blade displacement to obtain the Axial Velocity-Density Ratio (AVDR) and loss coefficient such that small variations in supply conditions during the survey have little effect. The AVDR was calculated using the definition

$$
\text { AOVR }=\frac{\int_{0}^{s} p_{2} V_{2} \cos B_{2} d s_{2}}{\int_{0}^{s} o_{1} V_{1} \cos B_{1} d s_{1}}
$$

and the loss coefficient using the definition

$$
\bar{\omega}=\frac{\bar{P}_{t 1}-\bar{P}_{t 2}}{P_{t 1}-P_{1}}
$$

where bars denote mass-averaged values.

Defining local-to-reference mass flux as

$$
k=\frac{\rho V \cos B}{\rho_{\text {ref }} V_{\text {ref }}}
$$

and local-to-reference pressure as

$$
C r=\frac{P}{P_{\text {ref }}}
$$

then Eqs. (1) and (2) become, respectively,

$$
\text { AVDR }=\frac{\int_{0}^{s} k_{2} d s_{2}}{\int_{0}^{s} k_{1} d s_{1}}
$$

and

$$
\bar{w}=\frac{\int_{0}^{s} k_{1} c r_{t 1} d s-\frac{1}{A V D R} \int_{0}^{s} k_{2} c r_{t 2} d s}{\int_{0}^{s} k_{1} c r_{t 1} d s-\int_{0}^{s} k_{1} C r_{1} d s}
$$

which is, equivalently,

$$
\bar{\omega}=\frac{\bar{C} r_{t 1}-\left(\frac{1}{A V D R}\right) \bar{C} r_{t 2}}{\bar{c} r_{t 1}-\bar{c} r_{1}}
$$

Probe data were reduced using Eq.(3) to (6), using over lapping quadratic interpolation to carry out the numerical integrations to obtain AVOR and loss coefficient.

Surface pressures were reduced to pressure coefficients using the definition

$$
C p=\frac{p-p_{1}}{\frac{1}{2} \rho_{1} V_{1}^{2}}
$$

To verify accuracy, the reference upstream conditions were derived in three different ways, namely: as the mass average of the blade-to-blade probe surveys; from the ensemble average of stagnation pressure readings and derived static pressure values during olade-to-blade probe surveys; and as derived from the fixed Prandtl probe measurements.

\section{CALCULATION METHOOS}

\section{Inviscid Flow Solution}

The inviscid flow about the blade section in the two-dimensional, blade-to-blade plane has been calculated by two methods: the method described by Katsanis, TSONIC (4), and the panel method developed by McFarland (17). The TSONIC program solves the stream function equation by finite difference techniques for the subsonic, compressible flow regime. It is necessary to specify as input the fluid properties, inlet total temperature and density, weight flow, blade geometry, inlet and outlet flow angle, finite difference mesh, and a meridional distribution of streamtube height. In the work presented herein, a linear distribution of streamtube height was utilized so as to match the measured axial velocity-density ratio.

Because the nature of the equations dictates that" the solution be of the boundary value type; the outlet flow angle must be specified on the downstream boundary. In these calculations, measured exit angle was used.

The TSONIC code uses a uniform mesn and, therefore, mesh packing in regions where more definition is desired is not permitted. To better define the calculated flow properties in the leading edge region, McFarland's panel code (17) was used. The code is a surface singularity method which solves the inviscid, irrotational, compressible blade-to-blade flow equations on a surface of revolution. Streamsheet thickness can be incorporated as a function of meridional distance. The governing equations are linearized by approximating compressibility effects, and solved using an integral technique (panel method). Up to 98 panel elements are possible. Computational time for a typical case is under $4 \mathrm{CPU}$ seconds on an IBM 3033 computer. The method is limited to subsonic flow and is less accurate for. low solidity blade rows. The same input data set used for TSONIC can be used for the panel code. Because of user freedom. to distribute panel elements, excellent definition in the leading edge region can be obtained.

Boundary Layer and Loss Calculations

Blade surface boundary layers were calculated using the program developed by McNally (5). In addition to the surface velocities, required input includes upstream flow conditions, fluid properties, and blade surface geometry. Among the output provided Dy the program are the conventional integral thicknesses, form factors, wall friction coefficient, and momentum thickness Reynolds number.

The program uses integral methods to solve the twodimensional, compress ible laminar and turbulent boundary layer equations in an arbitrary pressure gradient. Cohen and Resnotko's method (18) is used for the laminar boundary layer, transition is predicted by the Schlichting-Ulrich-Granville method (19), and Sasman and Cresci's metnod (20) is used for the turbulent boundary layer.

A Doundary layer which is initially laminar may proceed through normal transition to a turbulent boundary layer, or it may undergo some form of laminar separation before becoming turbulent. To provide flexibility for analyzing this behavior, several program options are available to the user. The calculations may proceed from a laminar boundary layer 
througn transition to a turbulent boundary layer. However, if laminar separation is predicted before transition, the turbulent calculations may be started by specifying a factor by which the last calculated value of momentum thickness is multiplied (this factor is commonly chosen to be 1.0 to satisfy conservation of momentum). This new momentum thickness and a value for form factor based on the last calculated momentum thickness Reynolds number are used as initial values for the turbulent calculations. In no case is an initial turbulent boundary layer allowed which has a momentum thickness Reynolds number less than 320 (21). Laminar separation is predicted when skin friction becomes zero. Prediction of turbulent separation is inexact. A separation criterion common to compressor blade analyses which use integral boundary layer methods is the incompressible form factor, Hi. Values of 1.8 to 2.6 nave been proposed and used in the past (22). In this report, critical values will be inferred from comparisons with data.

Total pressure loss coefficients were calculated using Stewart's method (23). Required input includes upstream flow conditions, fluid properties, displacement and momentum thickness at the trailing edge, blade spacing, trailing edge thickness, and exit velocity and flow angle. There is no satisfactory method for calculating loss when the boundary layer is separated, and a combination of analytic art and empiricism is generally used. In this report, for off-design cases where turjulent separation occurs, the displacement and momentum thicknesses at the location of critical form factor are assumed to remain constant to the blade trailing edge. These values are then used in the loss calculations from that point. Expected accuracy diminishes as incidence angle increases or decreases significantly from the design point.

\section{RESULTS AND DISCUSSION}

\section{Experimental 0bservations}

Since the cascade facility is somewhat unconventional, the quality of the flow is discussed before results are presented.

Flow quality. Data were obtained at in let air angles ranging from 25 to $46^{\circ}$. Care was taken at each setting to verify that the inlet pressure and velocity, and the outlet pressure were acceptably uniform, and that two-dimensional flow conditions with streamline contraction prevailed to the downstream measurement plane. Surveys of upstream and downstream flows for all test conditions are documented in detail in Ref. 14. In Figs. 6 and 7 are shown, for illustration, the results of spanwise and blade-to-blade probe surveys, respectively, at the design air-inlet angle of $39^{\circ}$. In Fig. 6 it can be seen that the flow remains nearly uniform over the center 40 percent of the blade.

In Fig. 7, showing the blade-to-blade conditions, the variation which appears aimost as noise on the inlet stagnation pressure profile is what remains of the wakes produced by the turning vanes. The peak-topeak variation seen in Fig. 7 is about +1.4 percent of the reference dynamic pressure, or +1.1 percent of the cascade inlet dynamic pressure. Thus, the inlet velocity was uniform to within 1.2 percent, and these small variations were accounted for by mass-averaging in evaluating both AVDR and losses. The inlet flow angle was uniform to within $0.5^{\circ}$ over the central four blade passages which were surveyed.

In viewing the downstream flow distributions in Fig. 7 , it should be noted that data were taken at close intervals $(0.25 \mathrm{~cm})$ for integration over one b lade passage, and over coarse intervals $(0.62 \mathrm{~cm})$ for the other three. All data points are shown connected oy straight lines. The minor departure from strictly repetitive conditions seen in the first (left-hand) blade interval was observed in all data sets. Integration to obtain blading performance was therefore carried out over the third blade interval. Periodicity within the blade passages was verified using corresponding pressure taps on three instrumented blades.

The quality of the test conditions did not change significantly except at the two extreme angles tested. At the lowest air angle $\left(25^{\circ}\right)$, the inlet uniformity decreased fractionally as a result of operating the inlet turning vanes considerably off design, while the physical separation of the wakes was increased because of the less oblique wall angle. At the highest angle $\left(46^{\circ}\right)$, the uniform core of the spanwise profile was reduced from 40 to 20 percent of span as a result of increased wall and passage viscous effects associated with increased loading.

Overall performance. The results obtained for the cascade performance based on survey probe measurements are snown in $\mathrm{Fig}$. 8. Also shown for comparison are the corresponding curves for data obtained by Himes (12) for the equivalent cascade of DCA blades. Some differences are noted. First, the loss coefficient for the controlled diffusion blade was measured to be less than that for the DCA Dlade at the design point, and over the useful range of air inlet angle. The data for loss coefficient were well behaved and showed no discernible effect of Reynolds number, except (perhaps) at the nighest air inlet angles.

Tnere were measurable differences in behavior at air inlet angles less than design. The outlet air angle did not change significantly for the CD blade, and ranged from $1 / 2$ to $3^{\circ}$ greater than measured for the DCA blade. Larger values of AVDR were also measured, altnough at angles approaching and exceeding the design value, the AVDR was measured to be lower than for the DCA cascade.

The AVDR is largely a consequence of the behavior of the end wall boundary layers in passing through the cascade, and the reason for the high value at $33^{\circ}$. inlet air angle is not clear. However, a much reduced static pressure rise was also registered, and this was confirmed by manometer readings of the distributions of wall static pressures. In fact, the pressure rise across the cascade dropped very abruptly with reduced air inlet angle, the drop being associated with the aetection of leading edge separation on the pressure side of the blade. Subsequent examination of all the information obtained in the tests pointed to a need to obtain more information in the range of inlet flow angles between 30 and $36^{\circ}$.

Vortex Shedding. When testing at the three lowest air inlet angles, pure, highly audible tones were heard as the tunnel was brought up to the required test conditions. The tones occurred at discrete settings of the plenum pressure, and the frequency increased (1080, $1960,2380,2790,3080 \mathrm{~Hz}$ ) as the inlet velocity at which it occurred increased (approximately $30,34.5$, $42,48.5,56 \mathrm{~m} / \mathrm{sec}$ ). Vortex shedding in the blunt blade wake was suspected when the Strouhal number based on trailing edge thickness was found to be 0.17 (when the tones occurred). An experiment was conducted to verify this explanation. The bluntness was removed by taping metal shim-stock to produce sharp trailing edges on all blades. The tones then did not occur. The removal of the shim-stock from one blade resulted in the reoccurrence of tones, but very faintly. 
Vortex shedding in turbomachinery blade wakes has been observed or suspected in several turbomachinery research experiments (24 to 28). However, the occurrence in the present case was particularly graphic Decause the frequencies were in the audible range and, despite the tunnel background noise level, some had the clarity of pure organ tones.

In high speed compressors and turbines, these reso$n$ ant frequencies $c$ an be over $100 \mathrm{kHz}$ and therefore too nigh to be detected by other than the highest response transducers (see Ref. 28, for example). The issue of vortex shedding must be raised when attempting to obtain comparisons of data with analyses which aim at the correct modeling of the flow. Whether any special treatment of the trailing edge flow is required when vortex sheoding occurs; compared to when it does not, requires that a more detailed study be conducted than was possible in the present series of tests.

\section{Comparison of Calculated and Experimental Results}

Inviscid flow computations, presented as static pressure coefficient versus percent of chord are compared with experiment in Fig. 9. The solid line represents panel code calculations and the dashed line represents TSONIC results.

There is very little difference between TSONIC and panel code calculations, except in the trailing edge region. Even there, the differences are minor. and are due to differences in the way each code models the trailing edge. No inviscid code will calculate the flow accurately near the trailing edge of a blade row because real, viscous effects are the most prominent in tnat region. For blades with round trailing edges, and particularly for the present case with a blade having a relatively large trailing edge diameter, the Kutta conaition does not truly apply. Some artifice must be used, and code developers employ different methods.

In TSONIC, Katsanis has used a mass injection or wake simulation model (1) sketched in Fig. 10. Tangents are formed at the intersection of the trailing edge circle and the blade surface, and extended to the vertical line which forms a tangent with the trailing edge circle (Fig. 10). The "wake" is then extended downstream with an orientation determined by the downstream whirl boundary condition. Pressure is allowed to vary across the simulated wake. This modelling removes the trailing edye circle and the attendant accelerations and decelerations associated with curvature. The user may vary exit angle until "closure" of the pressure aistribution occurs at the trailing edge to simulate a Kutta condition, or the user may specify an exit angle (such as an experimentally measured angle) and accept the crossing of suction and pressure surface pressures near the trailing edge, as in the present calculations. MCFarland sets the downstream static pressure by continuity considerations. He then forces the surface pressures on both surfaces to meet this pressure at the trailing edge by an interpolation process involving the last tnree panel elements on each surface. To achieve satisfactory results in the trailing edge region, it was found necessary in these calculations to use all 98 panel elements, and to pack points in the trailing edge (FTE set equal to 1.05).

Both methods are artificial. But inviscid flow, in itself, is artificial, and comparisons will always differ from the real flow in regions where viscous effects are strong.

Experimental measurements are generally in good agreement witn both methods of calculation. Viscous effects near the trailing edge begin to appear (as a divergence between calculation and measurement) at the design point (Betal $=38.91$ ) and increase as incidence increases. The last pressure tap on each surface is in a region of curvature and reflects the decreased pressure (increased velocity) there, particularly at lower inlet angles where suction surface boundary layers are relatively thin. Corresponding trailing edge accelerations do not appear in the calculations because the trailing edge has been modeled to remove them.

Measured pressures compare very well with the calculations in the leading edge region. Because of mesh limitations, TSONIC did not pick up any leading edge acceleration/decelerations upstream of 3 percent chord, and therefore was quite limited in boundary layer calculations. The panel code provided quite adequate resolution. A point of concern to code users has always been the calculated leading edge pressure spikes, and they are evident here even at the design point (Betal = 38.91). The excursions can be large and are mathematically consistent. How much of such an excursion is actually felt by the real flow will determine the nature of the boundary layer behavior. It appears from the measured data that the flow does experience a large proportion of the calculated leading edge velocity diffusion.

Two principal boundary layer calculations were made, one set using data from low Reynolds number tests to compare against flow visualization experiments performed at low Reynolds number $(340000)$, and one set using calculated pressure distributions corresponding to higher Reynolds number tests $(670000)$. The latter results were used to calculate loss coefficient to compare against measured loss.

Cnina clay flow visualization experiments were run on the suction surface of the blade over the full range of incidence angles, but at a low Reynolds number to accomnodate drying times of the solvent. The results are presented in Fig. 11. The axial position at which laminar separation-turbulent reattachment, transition, and turbulent separation is plotted against the inlet flow angle condition. The figure shows the existence of a laminar boundary layer to about 60 percent of chord at the two lowest flow angles. As incidence angle was increased, an abrupt change occurred and a laminar separation with turbulent reattachment was produced very near the leading edge ( 2 to 5 percent chord) at all of the nigher inlet angles.

The boundary layer calculations were made using experimental surface pressures as input, and results are also plotted on Fig. 11 . In all cases, laminar separation with turbulent reattachment was predicted, rather than pure transition. The calculated laminar separation point is reasonably close to experiment for Betal $=28.00$, but disagrees by about 15 percent chord at Betal $=32.87$. The calculated trends appear to be more valid because as incidence angle is increased, the adverse pressure gradient on the suction surface becomes steeper. Since laminar separation is strongly influenced by pressure gradient, earlier separation would be expected at Betal $=32.87$. At the higher inlet angles there is good agreement between experiment and calculation. The adverse gradient near the leading edge is very steep and produces almost immediate laminar separation. Due to the magnitude of the adverse pressure gradient in most cases, and the Reynolds number levels, the laminar separation bubbles are presumed to be short.

The experimental static pressure distributions already reflect turbulent separation, i.e., reduced adverse pressure gradients in the separated region near the trailing edge. Using these reduced gradients as input to a boundary layer calculation is not likely to result in prediction of turbulent separation, and did not for these calculations. It should also be noted that the experimental turbulent separation. locations shown in Fig. 11 for Betal $=28,32$ and $36^{\circ}$ are $\cdot$ more 
likely to be a localized drying phenomenon than a separation.

Boundary layer calculations on both surfaces were conducted for the highest Reynolds number case over the full range of incidence angles using calculated surface pressures as input. The calculated laminar and turbulent separation locations are shown on Fig. 12, using the china clay results as reference. Predicted laminar separation locations occur earlier for both Betal $=28$ and 32.87 , but agree with china clay results at the nigner inlet angles. No turbulent separation is predicted for the two lowest inlet angles, but is predicted for all inlet angles $38^{\circ}$ and greater. Assuming separation to occur at critical incompressible form factors of $2.0,2.2$, and 2.5 , the corresponding locations are plotted as percent chord in Fig. 12. The best correlation witn flow visualization data is given by $\mathrm{Hi}=2.2$. For the steep adverse gradient at Betal $=45.96$, a flow visualization separation location of 90 percent chord does not appear consistent. The 73 percent chord value, corresponding to $\mathrm{Hi}=2.2$ agrees better with the flattening of the pressure distribution shown in Fig. $9(f)$.

Total pressure loss coefficients were calculated using Stewart's method (23), and are compared with experimentally measured values in Fig. 13 . Attempting to calculate loss at off-design conditions using an integral boundary layer method and a one-dimensional loss model is a dubious enterprise. The calculations obviously break down at the two extremes of inlet angle (solid line). But agreement is remarkably good at the other incidence angles. For cases in which turbulent separation is predicted, the values of displacement and momentum thickness corresponding to $\mathrm{Hi}=2.2$ were assumed to remain constant to the trailing edge of the blade where the loss model was applied.

One notable point of disagreement is at the design inlet angle of 38.91. A recalculation of the boundary layer beginning at about 4 percent of chord beginning with a laminar boundary layer produces loss predictions in agreement with measurements (dashed line, Fig. D). To accomplish this it would be necessary for the boundary layer to have relaminarized in the reacceleration region between 4 and 25 percent of chord (Fig. 9(d)). Although no evidence of this is evident from-the china clay experiments, this appears to be the only way the measured loss values could be reconciled with computations.

The boundary layer calculations for the lowest inlet angle (24.49) were particularly troublesome on the pressure surface due to the excessively steep adverse pressure gradient. Laminar separation followed by turbulent separation was predicted. The boundary layer calculations can be continued through modestly high form factors, and values of displacement and momentum thickness were obtained at the trailing edge. But, with such a steep pressure gradient, turbulent separation would not be unexpected, and due to the much relaxed pressure gradient at 30 percent of chord, turbulent reattachment could be envisioned. Using this speculative approach, a loss coefficient comparable to experimental measurements was achieved by reattaching the turbulent boundary layer at 30 percent chord with a displacement and momentum thickness equivalent to a fourfold increase over values at predicted turbulent separation (dashed line, Fig. 13). Using such methods to preaict loss at far off-design conditions is clearly speculative. More sophisticated modeling is required or, at least, an extensive data base is necessary to permit consistent empirical enhancement.

\section{SUMMARY OF RESULTS}

The mid-span section of a controlled diffusion stator was tested in a two.dimensional cascade. Measurements over a wide range of incidence angles were obtained. A quite acceptable minimum loss coefficient level of 0.0241 was measured, a value lower than than measured on the reference double circular arc blade. Operating range was slightly better than for the DCA blade.

Measurements of surface pressure compare well against results computed by the TSONIC and panel codes over the full incidence angle range. Integral boundary layer calculations using the McNally code showed generally good agreement with flow visualization data when measured surface pressures were used as input. When computed inviscid surface pressures were used as input, laminar separation was predicted with less accuracy. Correlations between computed boundary layers and flow visualization data produced a critical incompressible form factor value for turbulent separation of 2.2 for this set of data. Loss calculations using the Stewart loss model provided remarkably good agreement except, as expected, at the extremes of the incidence angle range.

\section{ACKNOWLEDGMENTS}

The authors would like to acknowledge the assistance of Eric McFarland for nis aid with the panel code, and Allan McGuire of The Naval Postgraduate School for his efforts in obtaining the china clay and other data. The experimental program was funded by NASA Lewis Research Center, and partially by Naval Air Systems Command, Code $310 E$.

\section{REFERENCES}

1. Sanger, N.L., "The Use of Optimization Techniques to Uesign Controlled-Diffusion Compressor Blading, " Journal of Engineering for Power, Vol. 105, Apri1 1983, pp 256-264.

2. Urasek, D.C., Gorre11, W.T., and Cunnan, W.S., "Performance of Two-Stage Fan Having Low-AspectRatio, First Stage Rotor Blading," NASA TP-1493, Aug. 1979.

3. Crouse, J.E., and Gorre11, W.T., "Computer Program for Aerodynamic and Blading Design for Multistage Axial-Flow Compressors," NASA TP-1946, Dec. 1981.

4. Katsanis, T., "Fortran Program for Calculating Transonic Velocities on a Blade-to-Blade Stream Surface of a Turbomachine," NASA TN D-5427, 1969.

5. MCNally, W.D., "Fortran Program for Calculating Compressible Laminar and Turbulent Boundary Layers in Arbitrary Pressure Gradients," NASA TN D-5681, 1970 .

6. Vanderplaats, G.N., "CONMIN a Fortran Program for Constrained Function Minimization, User's Manual," NASA TM $X-62282,1973$.

7. Vanderplaats, G.N., "COPES a Fortran Control Program for Engineering Synthesis," Naval Postgraduate School, 1980.

8. Moebius, R.C., "Analys is and Testing to Improve the Flow from the Plenum of a Subsonic Cascade Wind Tunnel," M.S. thesis Naval Postgraduate School, Monterey, CA, 1980.

9. Duval, David A., "Evaluation of a Subsonic Cascade Wind Tunnel for Compressor Blade Testing," M.S. thesis, Naval Postgraduate School, Monterey, CA, 1980 . 
10. Cina, Frank S., "Subsonic Cascade Wind Tunnel Tests Using a Compressor Configuration of DCA Blades," M.S. thesis, Naval Postgraduate School, Monterey, CA, 1981 .

11. Molloy, William D., "Preliminary Measurements and Code Calculations of Flow Through a Cascade of DCA Blading at a Solidity of $1.67, "$ M.S. thesis, Naval Postgraduate School, Monterey, CA, 1982:

12. Himes, Stephen J., "Report of Tests of a Compressor Configuration of DCA Blading," M.S. thesis, Naval Postgraduate School, Monterey, CA, 1983.

13. McGuire, Alan G., "Determination of Boundary Layer Transition and Separation on Compressor Blades in a Large Subsonic Cascade," M.S. 'thes is, Naval Postgraduate School, Monterey, CA, 1983.

14. Koyuncu, Y., "Report of Tests of a Compressor Configuration of CD Blading," M.S. thesis, Naval Postgraduate School, Monterey, CA, 1984.

15. Zebner, H., "Procedure and Computer Program for Approximation of Data (With Application to Multiple Sensor Probes), "Naval Postgraduate School Contractor Report, NPS67-80-001CR, Monterey, CA, 1980 .

16. Neuhoff, F., "Computer Software for the Calibration of Pneumatic and Temperature Probes, "Naval Postgraduate School, Turbopropulsion Laboratory Technical Note 82-03, Monterey, CA, 1982.

17. McFarland, E.R., "A Rapid Blade-To-Blade Solution for Use in Turbomachinery Design," J. of Engineering for Gas Turbines and Power, vol. 106, No. 2, April 1984, pp. 376-382.

18. Cohen, C.B., and Reshotko, E., "The Compressible Laminar Boundary Layer With Heat Transfer and ArDitrary Pressure Gradient," NACA TR-1294, 1956.

19. Scnlichting, H., "Origin of Turbulence II," Boundary Layer Theory, 7th ed., McGraw-Hill, New York, 1979, pp 489-554.

20. Sasman, P.K., and Cresci, R. J., "Compressible Turbulent Boundary Layer with Pressure Gradient and Heat Transfer," AIAA Journa 1, Vol. 4, No. 1, Jan. 1966, pp. 19-25.
21. Preston, J.H., "The Minimum Reynolds Number for a Turbulent Boundary Layer and the Selection of a Transition Device," J. of Fluid Mechanics, Vol. 4, iNo. 1 , Jan 1958, pp. 373-384.

22. Von Doenhoff, A.E., and Tetervin, N., "Determination of General Relations for the Behavior of Turbulent Boundary Làyers," NACA Wartime Report $\mathrm{L}-382,1943$.

23. Stewart, W.L., "Analysis of.Two-Dimensional Compressible-Flow Loss Characteristics Downstream of Turdomacnine Blade Rows in Terms of Basic Boundary-Layer Characteristics," NACA TN 3515 , July 1955.

24. Heinemann, H.J. and Butefisch, K. A., "Determination of the Vortex Shedding Frequency of Cascades with Different Trailing Edge Thicknesses," AGARD-CP277, 1977.

25. Heinemann, H.J., Lawaczeck, 0 . and Butefisch, K. A., "von Karman Vortices and Their Frequency Determination in the Wakes of Profiles in the Suband Transonic Regimes," IUTAM Symposium Transonicum II. Springer Verlag, Berlin/Heidelberg/New York, pp. 75-82, 1976.

26. Ng, W.F., and Epstein, A.H., "Unsteady Losses in Transonic Compressors," J. of Engineering for Gas Turbines and Power, Vol. 107, April 1985, pp. 345-353.

27. Hathaway, M.0., Gerts, J., Epstein, A. and Strazisar, A.J., "Rotor Wake Characteristics of a Transonic Axial Flow Fan," AIAA-85-1133, presented at the AIAA/SAE/ASME/ASEF 21st Joint Propulsion Conference, Monterey, CA, July 8-10, 1985.

28. Neuhoff, F., Shreeve, R.P., and Fottner, L., "Evaluation of the Blade-to-Blade Flow from a High Speed Compressor Rotor," Paper submitted for presentation at the 31st ASME International Gas Turbine Conference, Dusseldorf, June 1986.

TABLE I - BLADE AND CASCADE GEOMETRY

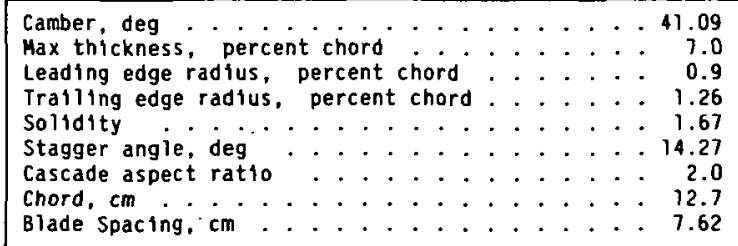


TABLE III - MEASUREMENT UNCERTAINTY

\begin{tabular}{|l|c|c|}
\hline \multicolumn{1}{|c|}{ Measurement } & Method & Uncertainty \\
\hline Probe position & Linear potentiometer & $\pm 0.02 \mathrm{~cm}$ \\
Flow angle $\left(B_{1}\right)$ & & $\pm .2 \mathrm{deg}$ \\
$\left(B_{2}\right)$ & $\begin{array}{l}\text { Angle potentiometer } \\
\text { Angle potentiometer }\end{array}$ & $\pm .5 \mathrm{deg}$ \\
Pressures. & & \\
Plenum & Scanivalve \& transducer & $\pm .05 \mathrm{~cm}$ water \\
Wall static & Scanivalve \& transducer & $\pm .05 \mathrm{~cm}$ water \\
Blade surface & Scanivalve \& transducer & $\pm .05 \mathrm{~cm}$ water \\
Probe & Scanivalve \& transducer & $\pm .05 \mathrm{~cm}$ water \\
Flow field & Calibrated probe & $\pm .05 \mathrm{~cm}$ water \\
Stagnation pressure & & $\pm .2 \mathrm{~cm}$ water \\
Static pressure & & \pm .5 percent \\
Velocity & &
\end{tabular}




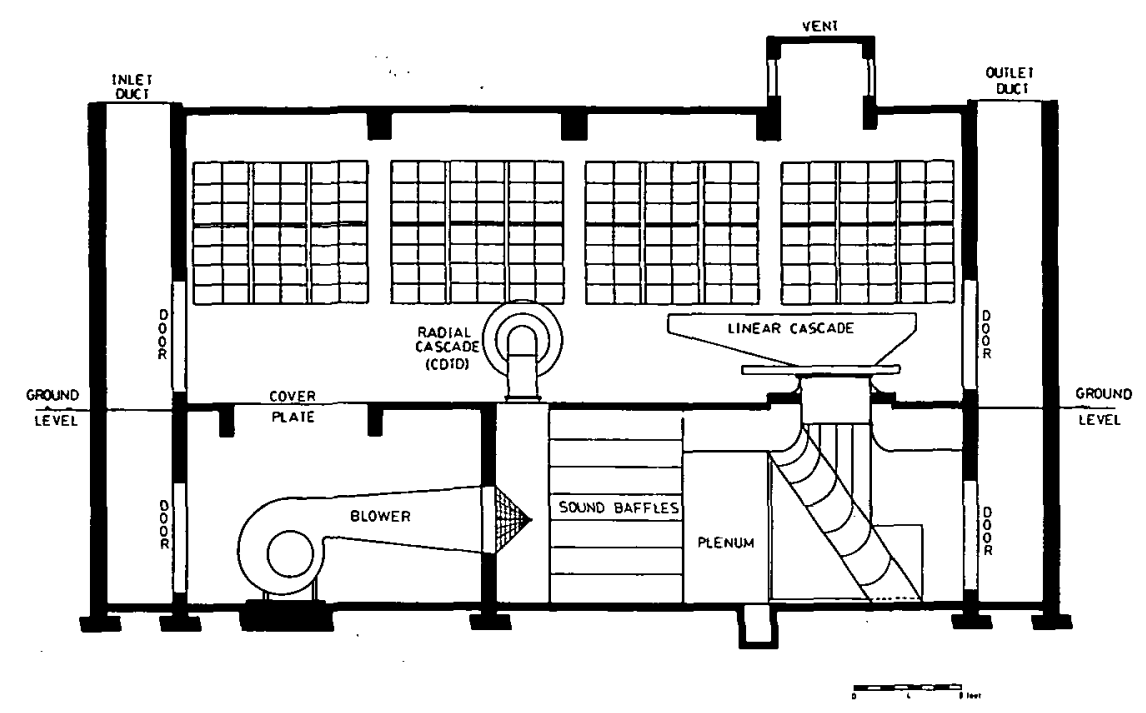

Figure 1. - Cascade wind tunnel test facility.

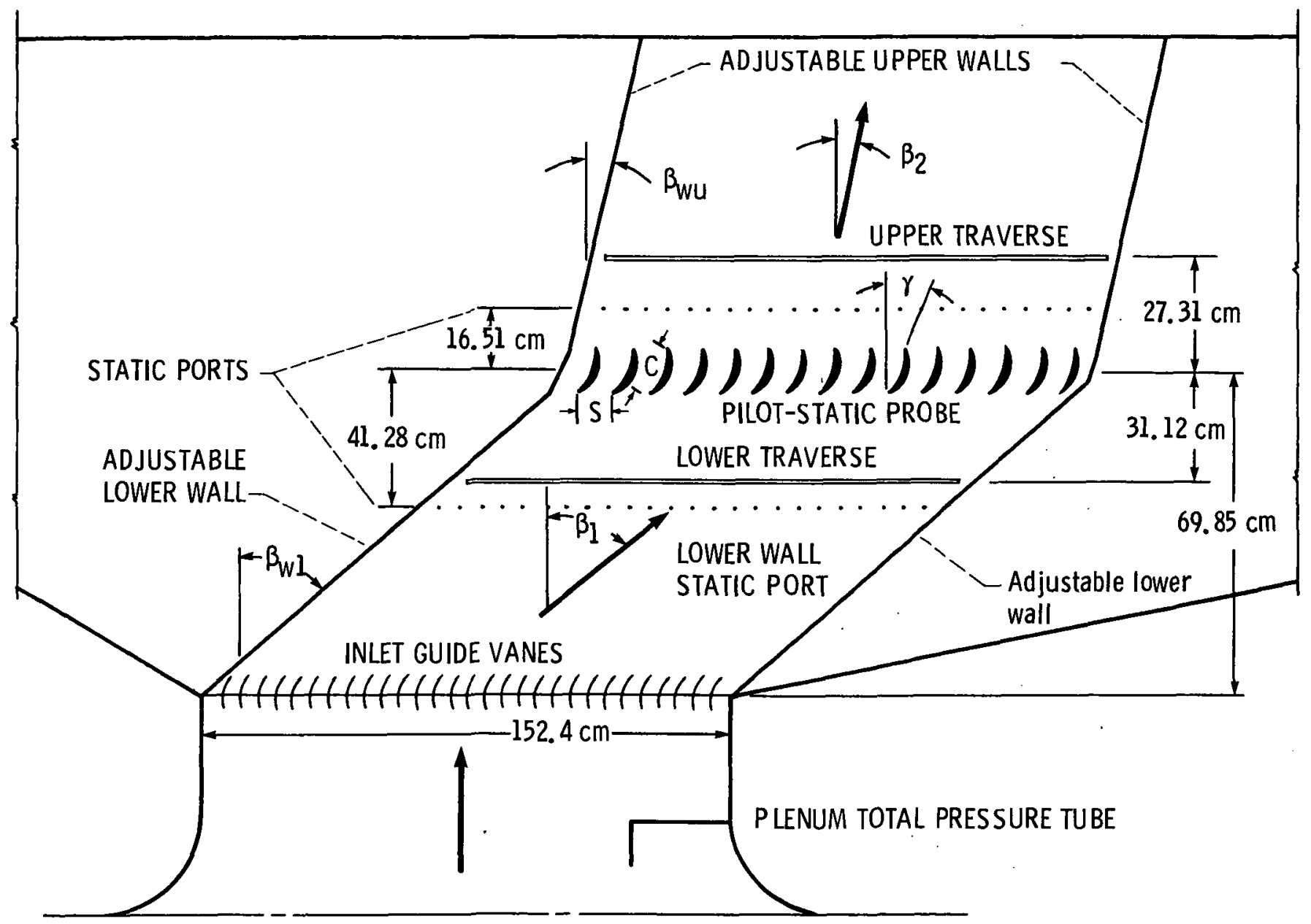

Figure 2. - Test section instrumentation and physical dimensions. 


\section{ORIGINAL PAGE IS \\ OF, POOR QUALITY}

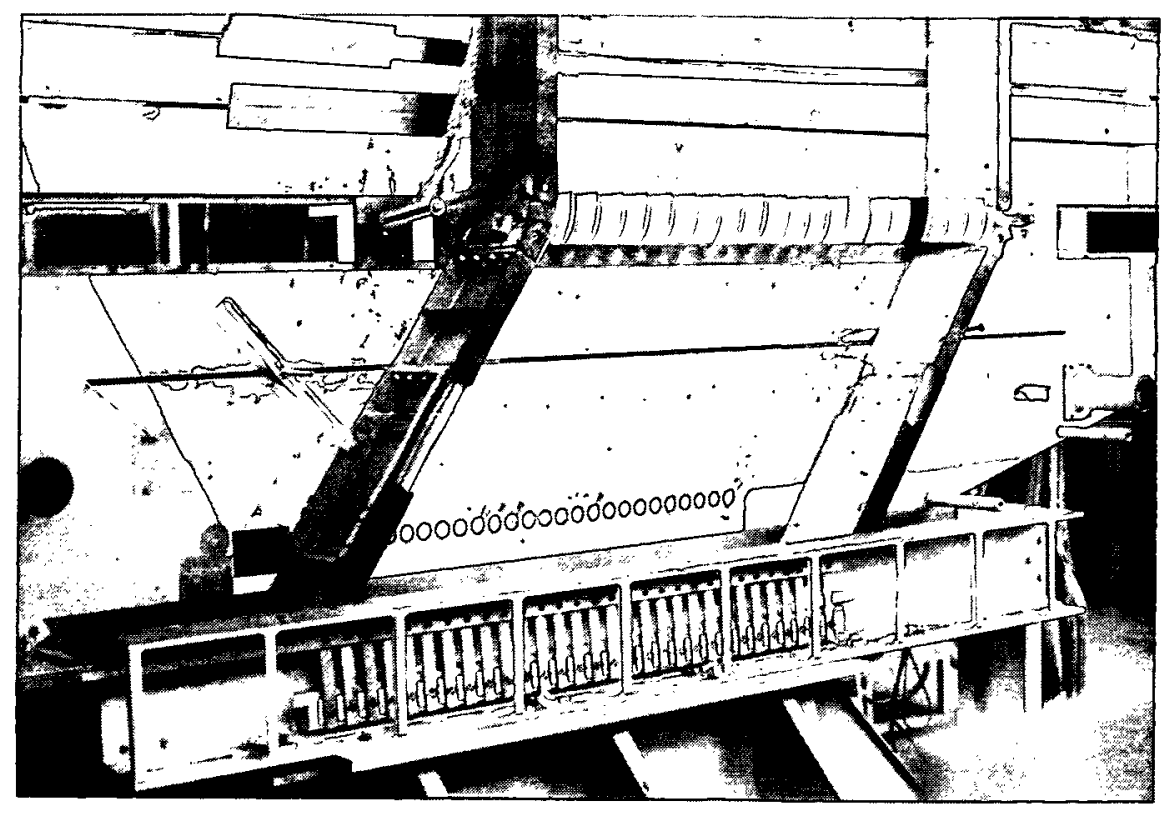

Figure 3. - Cascade test section with front wall removed.

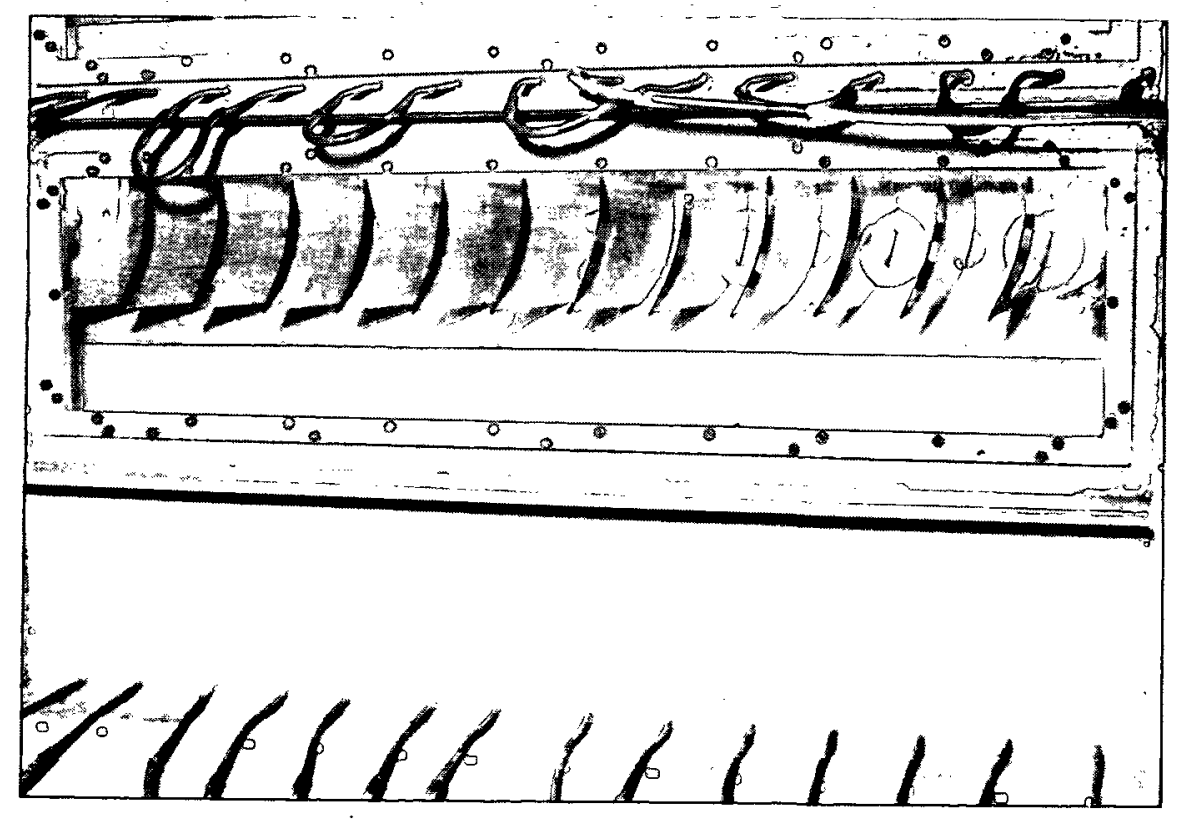

Figure 4. - View of test blading through plexiglas window. 


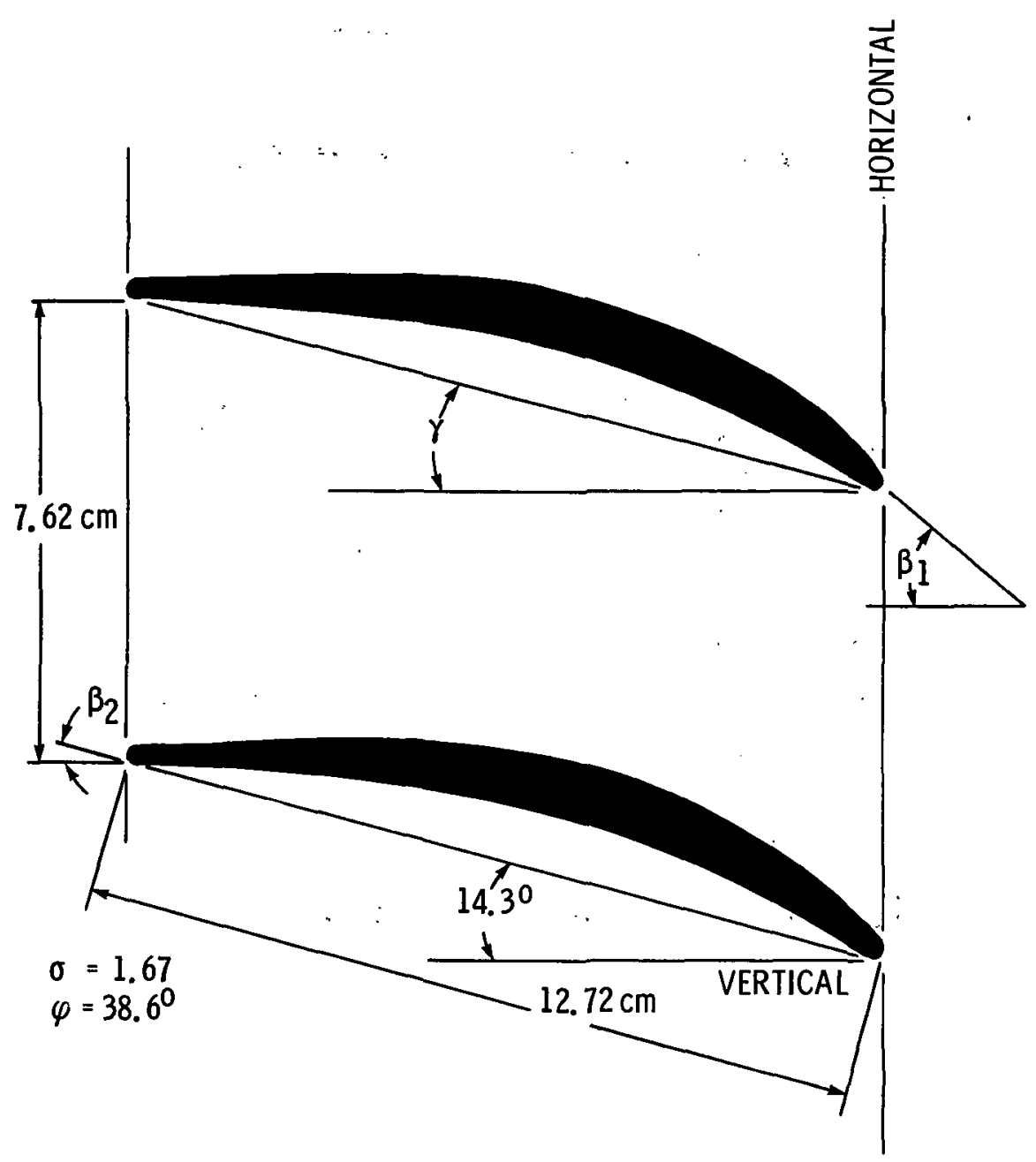

Figure 5. - Cascade geometry. 

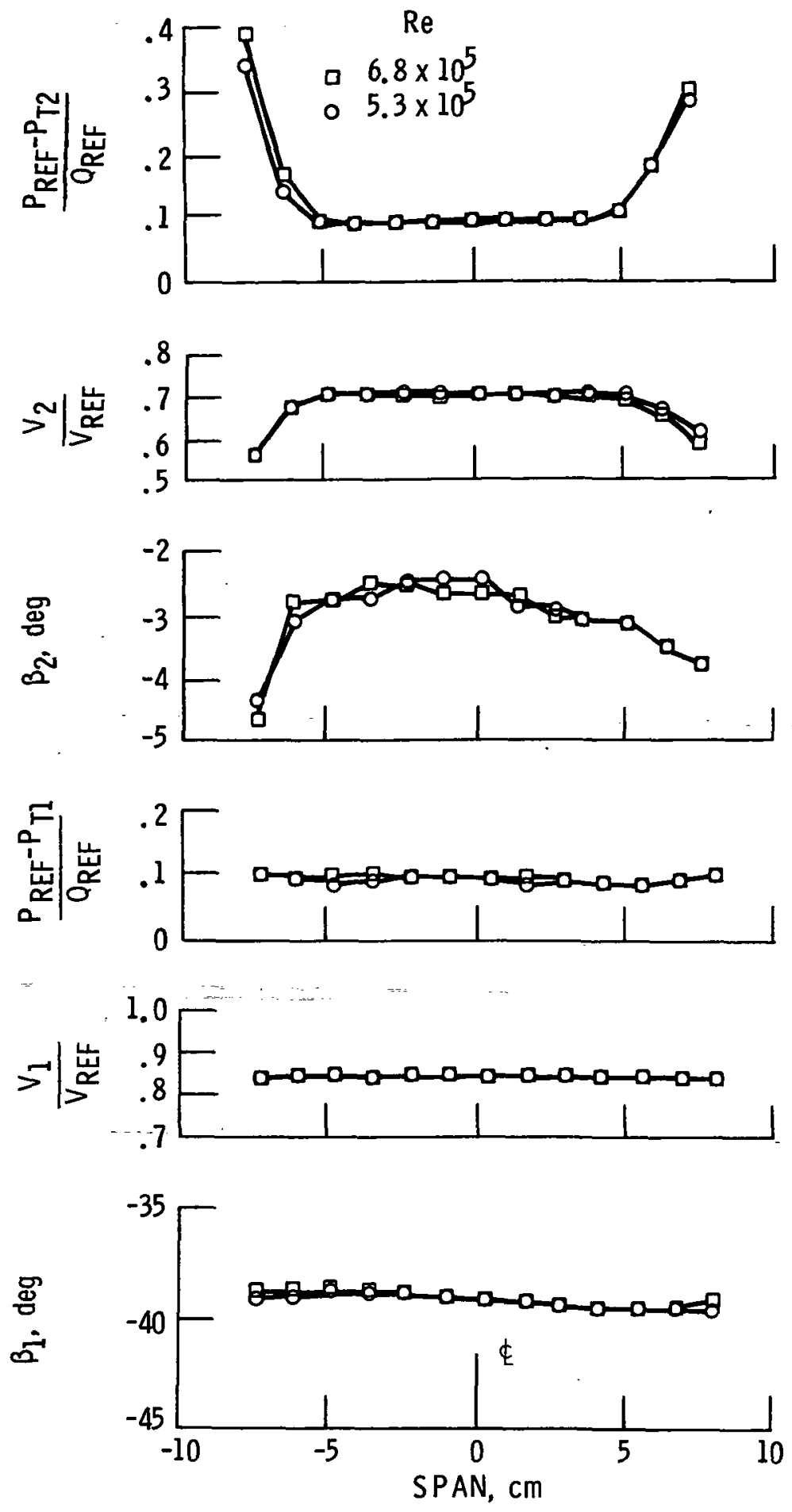

Figure 6. - Measured inlet and outlet flow field in the spanwise direction at design inlet angle. 

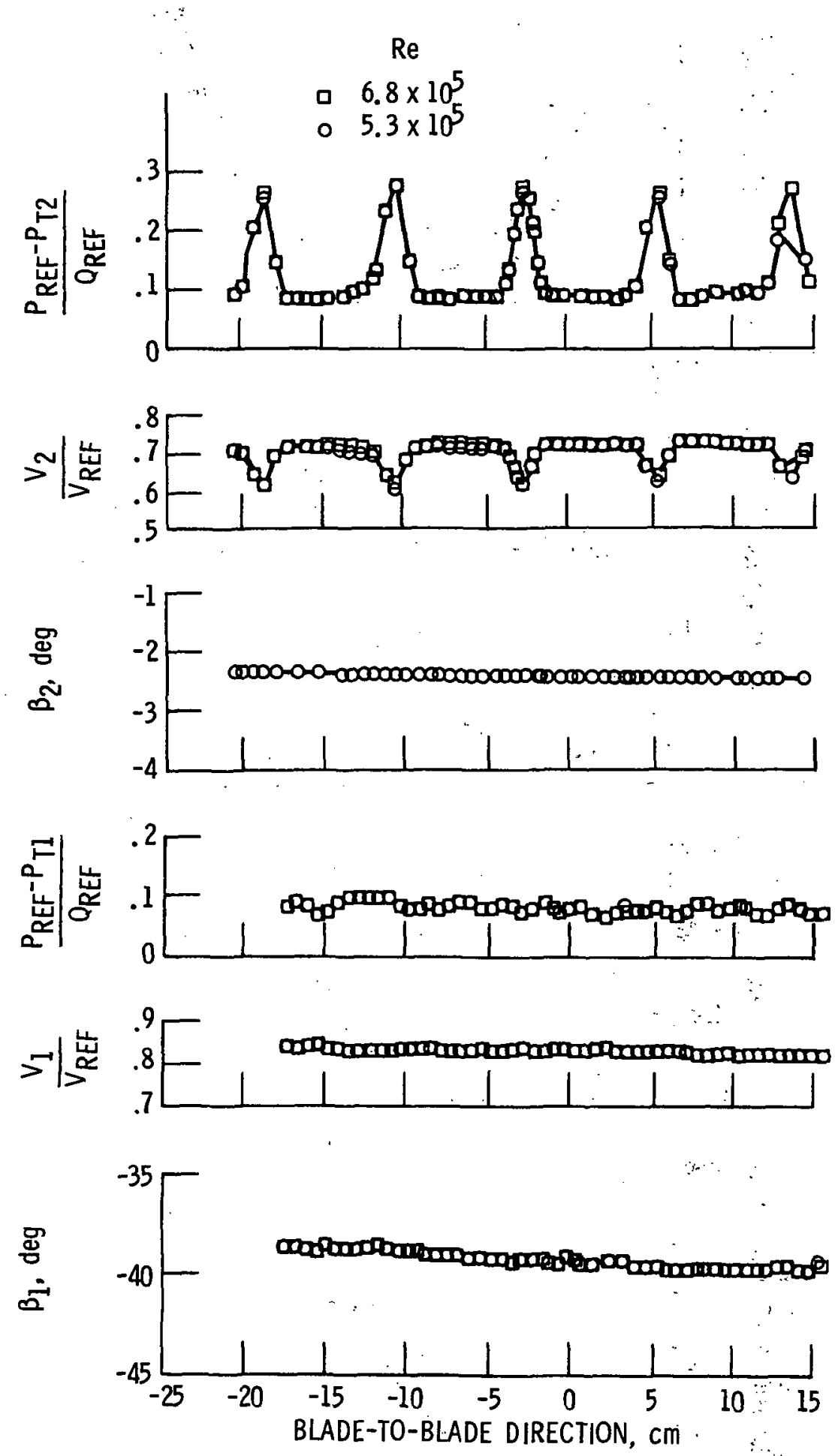

Figure 7. - Measured cascade inlet and outlet flow field in blade-to-blade direction at design inlet angle. : 

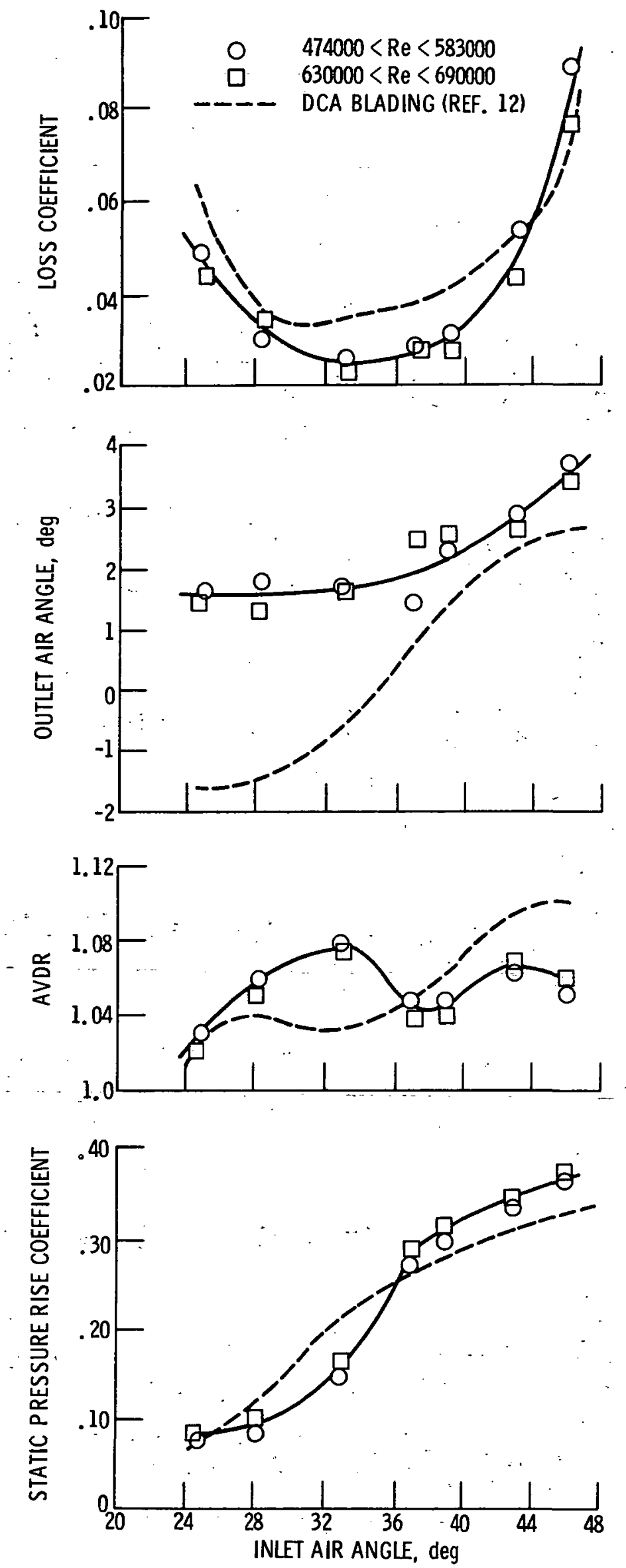

Figure 8. - Cascade performance from probe survey data. 


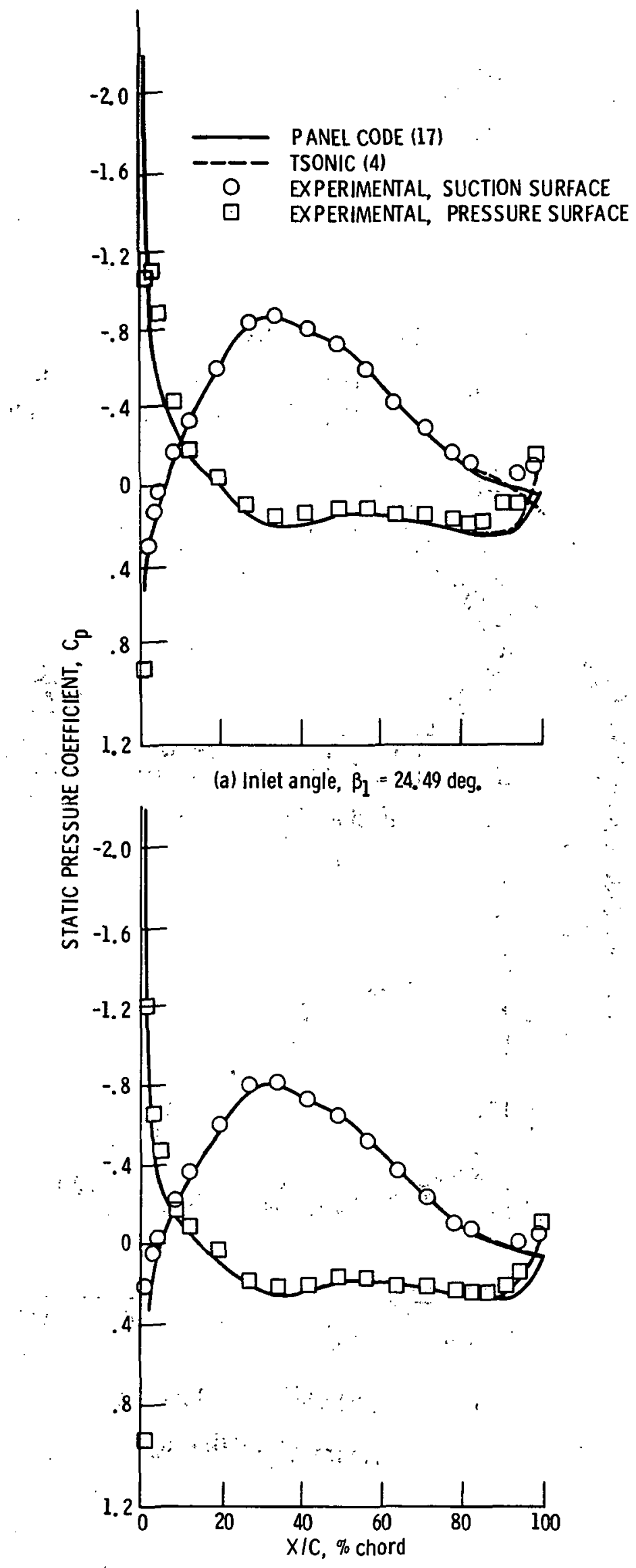

(b) Inlet angle, $\beta_{1}=28.00 \mathrm{deg}$.

Figure 9. - Comparison of experimental and calculated surface pressure coefficient. 


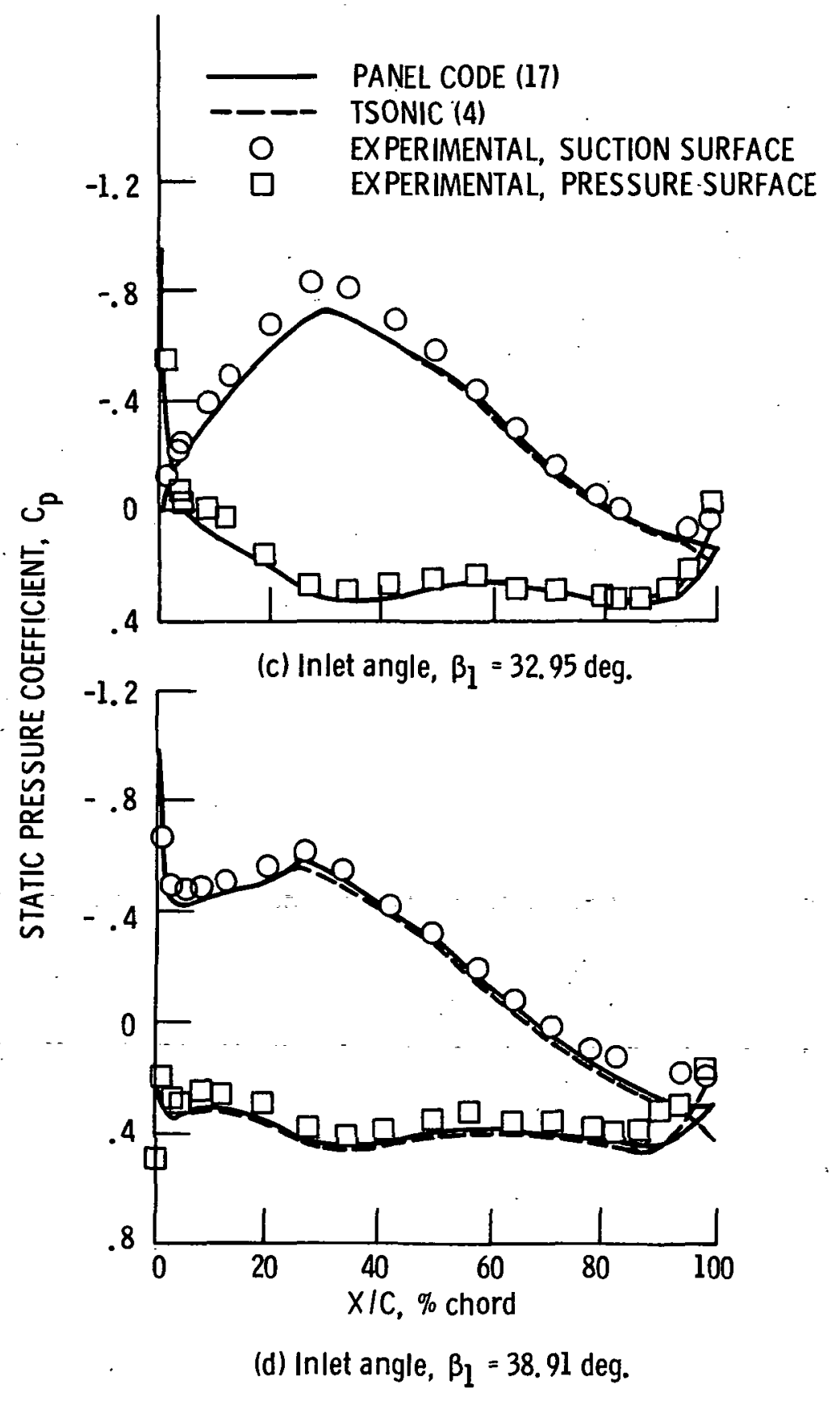

Figure 9. - Continued. 


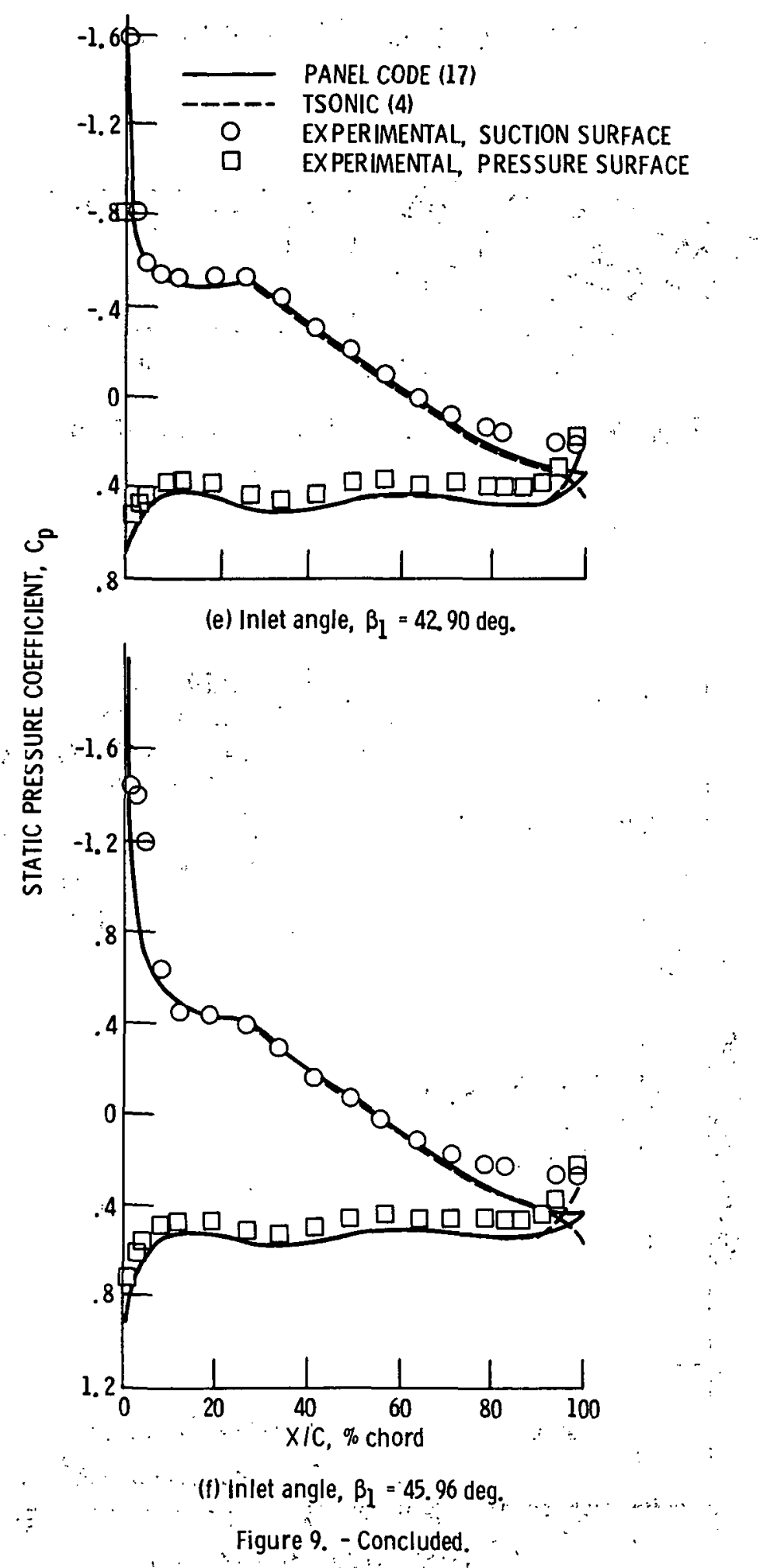




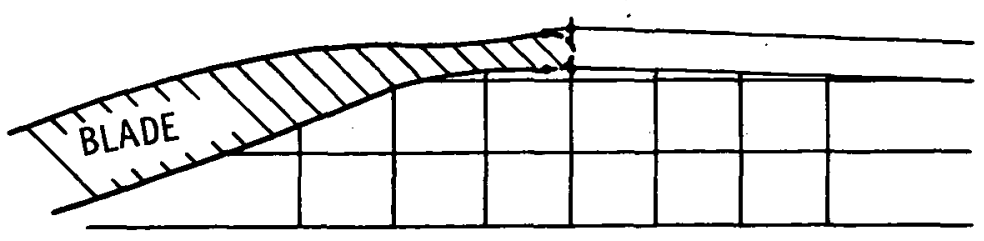

(a) Construction of effective wake.

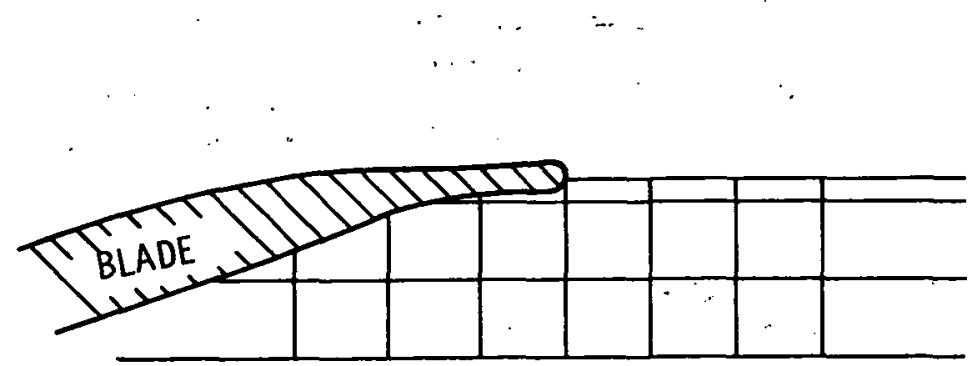

(b) Original trailing edge model (ref. 9).

Figure 10. - Mass flow injection model: TSONIC program.

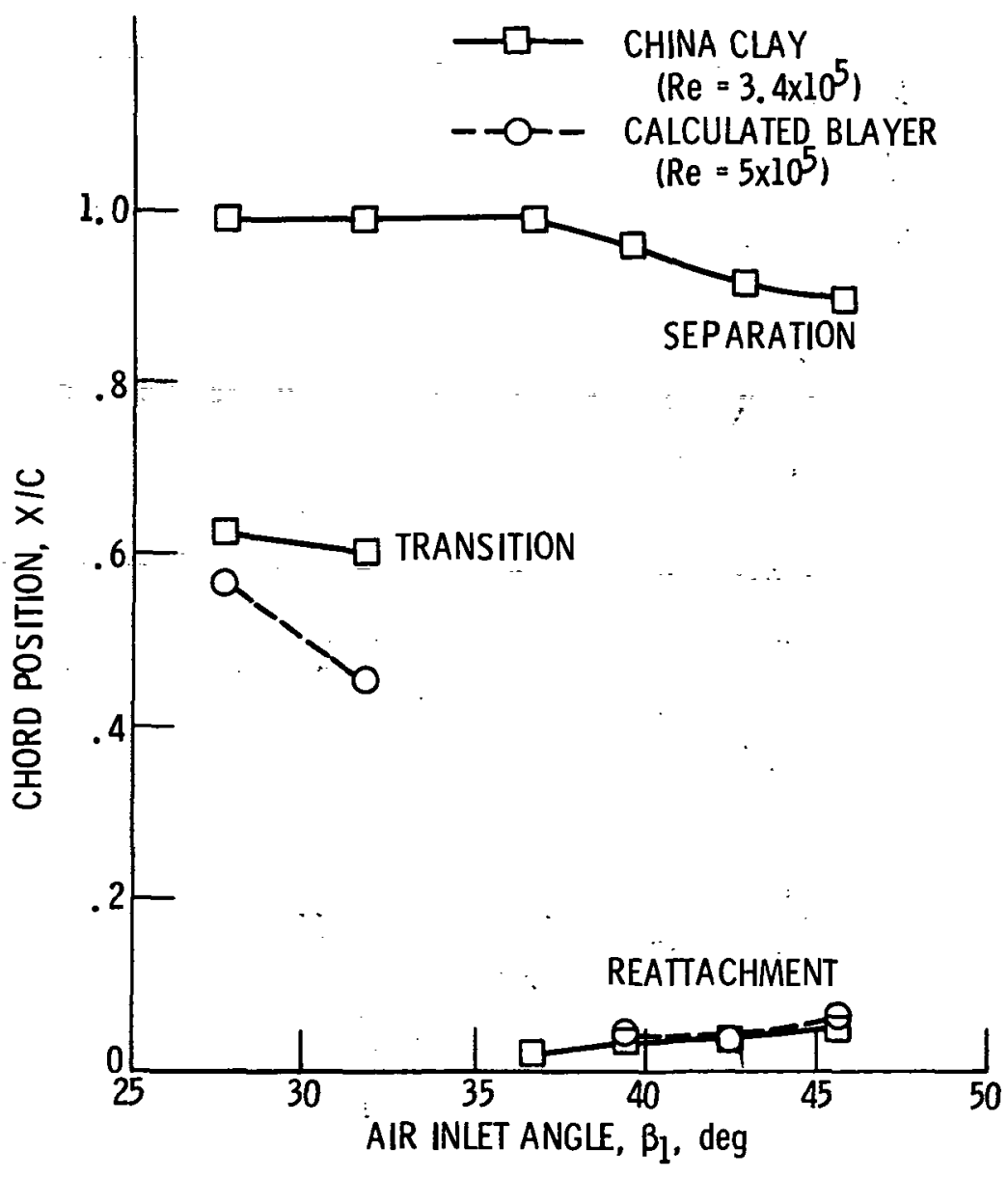

Figure 11. - Comparison of boundary layer behavior between China clay experiments and calculations using experimental surface pressure distributions. Suction surface. 


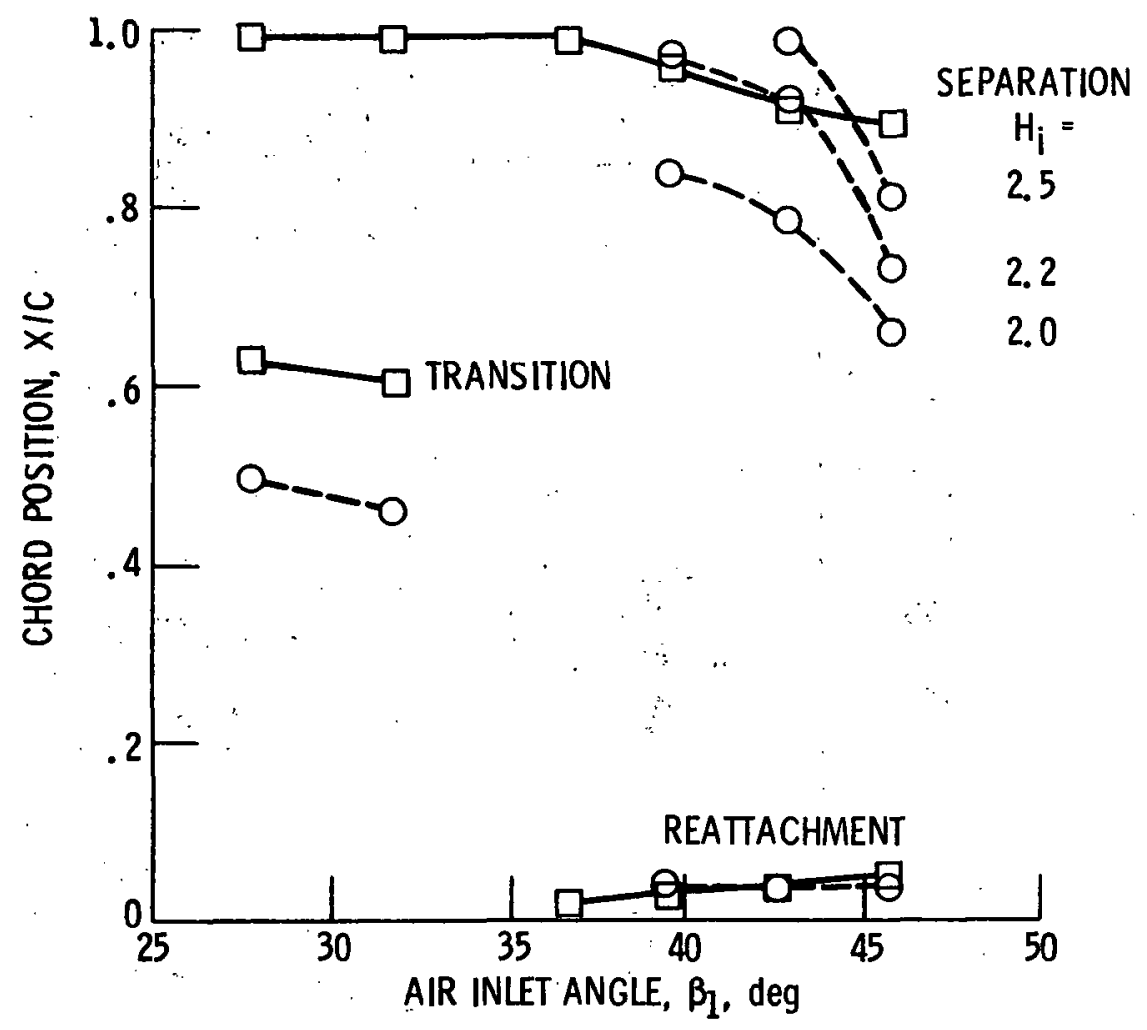

Figure 12. - Comparison of boundary layer behavior between China clay experiments and computations using calculated surface pressure distributions. Suction surface.

$$
\begin{gathered}
\text { CHINA CLAY } \\
\left(\operatorname{Re}=3.4 \times 10^{5}\right) \\
-0-\quad \text { COMPUTED } \\
\left(\operatorname{Re}=7 \times 10^{5}\right)
\end{gathered}
$$




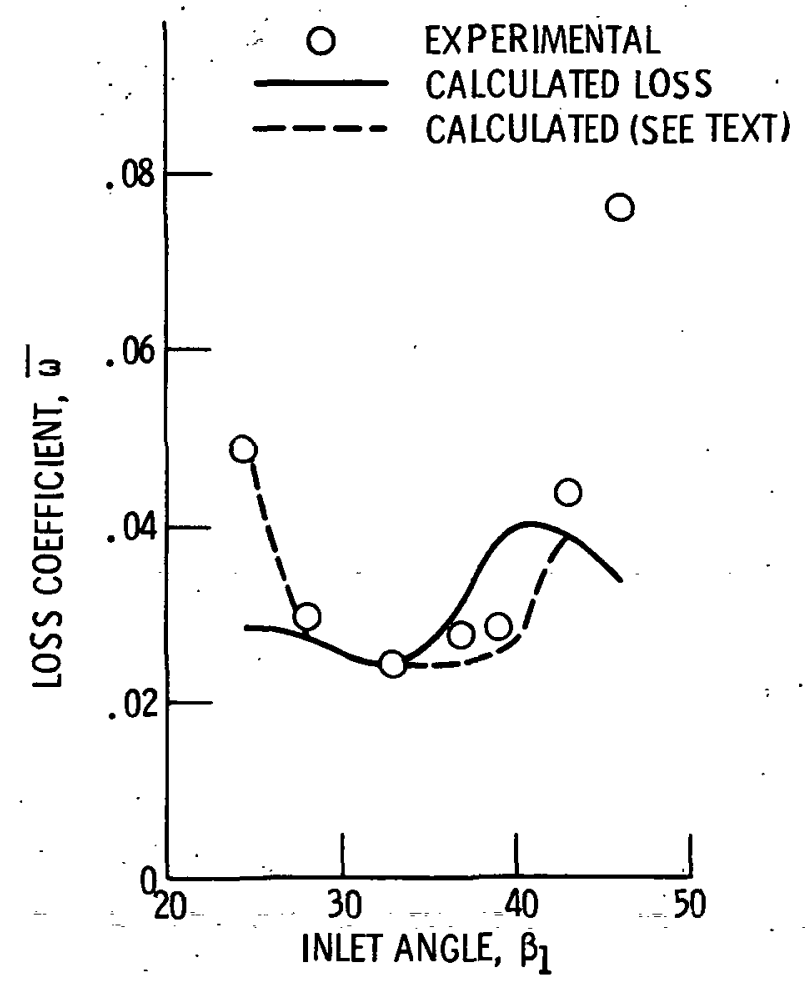

Figure 13. - Calculated loss (Stewart's model) compared with experimental measurements. 


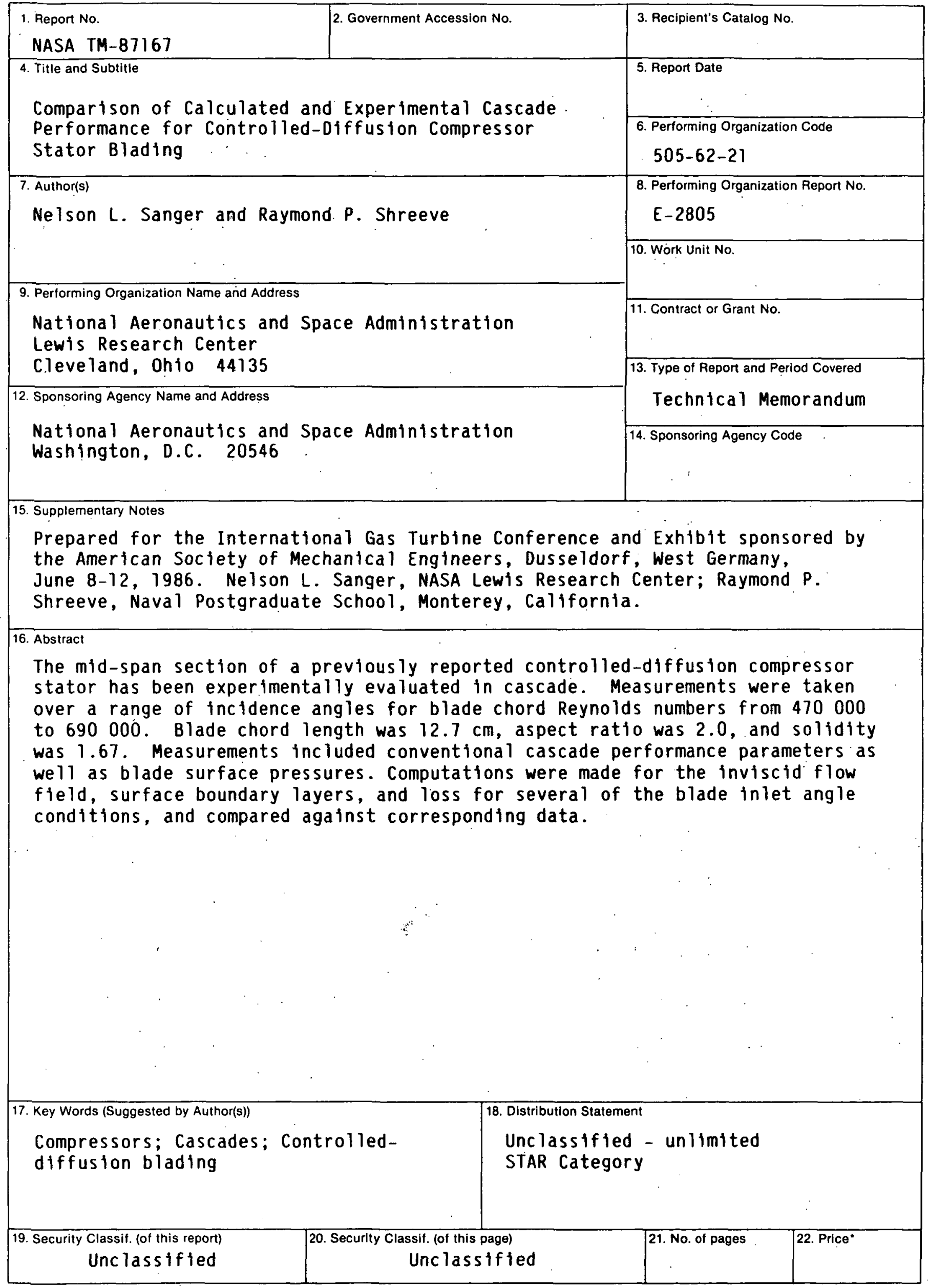


National Aeronautics and Space Admınistration

Lewis Research Center

Cleveland. Ohio 44135

\section{Official Business}

Penalty for Private Use $\$ 300$
SECONO CLASS MAIL

ADDRESS CORRECTION REQUESTED

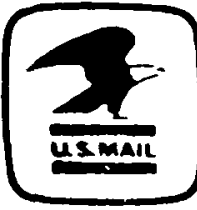

Poslage and Fees Paid National Aeronautics and Space Adminıstratıon NASA-451 